\title{
Maternity Nurses' Performance Regarding Cardiopulmonary Resuscitation During Pregnancy: Simulation Based Intervention
}

\author{
Aziza Ibrahim Mohamed ${ }^{1}$, Hemmat Mostafe Elbana ${ }^{1} \&$ Samah Abd Elhaleim ${ }^{1}$ \\ ${ }^{1}$ Maternal and NewbornHealth Nursing, Benha University, Egypt \\ Correspondence: Aziza Ibrahim Mohamed Hassan, Assistant professor of Maternal and Newborn Health Nursing, \\ Benha University, Egypt.
}

Received: March 5, 2018

Accepted: March 28, 2018

Online Published: April 2, 2018

doi:10.20849/ijsn.v3i1.356

URL: https://doi.org/10.20849/ijsn.v3i1.356

\begin{abstract}
Background: Training maternity nurse regarding cardiopulmonary resuscitation during pregnancy is most important for properly care of such emergency situations during pregnancy and save woman's and fetal lives. The aim of the present study was to evaluate effect of simulation-based intervention on maternity nurse' performance regarding cardiopulmonary resuscitation during pregnancy. Design Aquasi-experimental design was adopted in the current study. The study was conducted at woman's health nursing department training lab at faculty of nursing affiliated at Benha University Hospital. Where a lot of women are admitted for normal and vaginal delivery, gyneacological treatment and surgery.

Subjects: A Convenient sample of a total 52 maternity nurses was included.

Tools: Two tools: Were used for data collection. Interviewing questionnaire sheet was concerning with nurses personnel characteristics' and knowledge regarding cardiopulmonary resuscitation during pregnancy and performance check list sheet.the result of the present study that there was a highly statistical difference between knowledge and practical scores of maternity nurse at pre and post intervention. The study concluded that the simulation bases intervention is highly improved maternity nurses' performance regarding cardiopulmonary resuscitation during pregnancy. The present study recommended that simulation-based training regarding cardiopulmonary resuscitation should be provided for all obstetrics health care givers.
\end{abstract}

Keywords: simulation, maternity nurse, training program, performance, cardiac pulmonary resuscitation

\section{Introduction}

Cardiopulmonary arrest during pregnancy is life threatening condition and a leading cause of death. It presents a unique clinical scenario involving two patients: the mother and the fetus. The management of cardiac arrest in pregnancy is an important task for the emergency health team (Adam et al., 2016). The clinical outcome of mother or fetus will often depends on the successful resuscitation of the first few minutes. And a rapid multidisciplinary approach, including anesthesiology, medicine, obstetrics, neonatology, and sometimes cardiothoracic surgery. Basic and advanced cardiac life support algorithms should be implemented (Rittenberger, 2010).

The anatomic and physiologic changes during pregnancy result in significantly decreased cardiovascular and pulmonary reserves which complicate resuscitation. Cardiac output rises $30 \%$ to $50 \%$ as a result of increased stroke volume and to lesser extent increase in maternal heart Rate from 15-20bpm (Tan., 2013). Systemic vascular resistance decreased as a result of increase in several endogenous vasodilators including progesterone, estrogens and nitric oxide leading to decrease in mean arterial pressure, enlarge uterus can produce increased after load through compression of aorta and decrease cardiac return through the compression of inferior vena cava (Al-Foudri, 2010).

The estimates incidence of cardiac arrest during pregnancy ranged from $1 / 15,000$ to $1 / 30,000$-with maternal survival rates as low as $7 \%$ reported. The recently published scientific statement from the American Heart Association (AHA) addressing cardiac arrest in pregnancy is the first of its kind, even though a cardiac arrest risk in pregnancy has long been a known (Myerburg, 2015).

Cardiac arrest occurs only about one in every 30000 late pregnancies, but survival from such an event is exceptional. Most deaths are from acute causes, with many mothers receiving some form of resuscitation. 
However, the number of indirect deaths that is, deaths from medical conditions exacerbated by pregnancy is greater than that of deaths from conditions that arise from pregnancy itself (Micheal, 2014).

There are different causes of cardiac arrest during pregnancy; the most common cardiac cause is myocardial infarction, due to either premature atherosclerosis or coronary artery dissection. This relates in part to an increase in the proportion of pregnancies in women beyond the age of 35 years. The second most common cardiovascular cause is aortic aneurysms/dissections, and the third is the category of complex congenital heart diseases (Micheal, 2014).

The British center for maternal and child enquiries report has stated that timely recognition of pregnant women at risk of potentially life threatening conditions plays an important role in the appropriate institution of treatment (Wong et al, 2014).

Cardiopulmonary resuscitation during pregnancy is an integral part of health care of woman and fetus. Basic Life training (BLS) is highly essential for all healthcare staff members; especially to those who are working in obstetrics care units because protocol based management avoids confusion, wastage of time which save many lives. Survival after cardiopulmonary arrest is usually depends on early intervention, quality of cardiopulmonary resuscitation (CPR) and time to defibrillation (Demestiha et al., 2012).

Simulation is a technique for practice and learning that can be applied to many different disciplines and trainees; Simulation-based intervention is the way for developing health care provider knowledge, skills, and attitudes, in order to protect patients from unnecessary risks (Institute of Medicine, 2015).

The utilization of simulation to improve outcomes has been demonstrated in multiple domains because it gives the trainer the opportunity to experience uncommon scenarios, receive feedback, and perhaps most importantly correct mistakes before they become part of the learner's or groups mental model (Lavonas et al., 2015).

Simulation has also been shown to improve competencies in obstetric emergency decision-making, leadership, and individual and team performance This sentiment is reinforced in Committee Opinion Number 590 from The American College of Obstetricians and Gynecologists(ACOG), which states that one parameter for preparedness is integration of standardized emergency simulation and drills to identify and correct common clinical errors, to practice effective communication in a crisis, and to reinforce protocols, activation criteria, and critical interventions (Robertson et al., 2009).

Nurses are often first responders in cardiopulmonary arrest, and should be able to begin basic emergency care. Basic emergency care is crucial, rapid mobilization of expert resuscitation team and BLS performed competently until the arrival of these teams gives women the best chance for return of spontaneous circulation (Neal et al., 2012) Nurses play an important role in emergency management. Maternity nurse practice of resuscitation skills is best achieved through regular effective training. However, providing this teaching in a busy high-acuity maternity care units had become a challenge due to time and logistical constraints. A program to effectively and efficiently teach ICU nurses the skills necessary in patient resuscitation was developed using simulation training to better meet maternity learning needs (Jeejeebhoy et al., 2015).

\subsection{Aim of the Study}

The aim of the present study was to evaluate effect simulation based intervention on maternity nurses' performance (knowledge and practice) regarding cardiopulmonary resuscitation during pregnancy.

\subsection{Research Hypothesis}

Simulation based intervention will improve maternity nurses knowledge and practice regarding cardiopulmonary resuscitation during pregnancy.

\subsection{Significance of the Study}

However cardiac arrest is rarely occurring during pregnancy. It is associated with a high maternal and fetal mortality rate. A multi-center study 2 reported the outcomes of 55 women who experienced cardiac arrest during pregnancy, including 12 who underwent perimortem cesarean delivery: only $15 \%(8 / 55)$ survived. So a survival rate of pregnant women is very poor.

In addition the center for maternal and child enquiries (CMACE) reports, concerning 2006-2008, have revealed that care was substandard in more than $50 \%$ of maternal deaths caused by cardiac arrest and that resuscitation skills were "considered very poor in an unacceptably high number of cases" (CMACE 2011). Several studies published on this matter (Cohen et al., 2008. Einav, et al., 2008) have shown a significant lack of knowledge among obstetric care providers about differences in the resuscitation of the pregnant women. A variety of medical professionals are 
involved in the resuscitation of these women and, as cardiac arrest is such a rare occurrence on labor and delivery units, staff members are often unprepared to address this unexpected event.

So, providing high quality care quickly and efficiently to the pregnant patient who suffers cardiac arrest is a complex process, which requires previous awareness and training. From the clinical experience at the studied setting, the rdetention of cardiopulmonary resuscitation skills was poor. Particularly among maternity nurses who have little opportunity to practise CPR. Regular short periods of practice on a manikin are therefore essential. So the aim of the current was to evaluate effect of simulation based intervention on maternity nurses' performance.

\section{Subject and Methods}

\subsection{Research Design}

A quasi-experimental design was used in the present study.

\subsection{Research Setting}

Woman's health nursing department training lab at faculty of nursing affiliated at Benha university hospital.

\subsection{Subjects}

A convenient sample of a total 52 maternity nurses who working at obstetric and gyneacological department affiliated at Benha university hospital.

\subsection{Tools of Data Collection: Two Tools Was Used to Collect Data}

Tool I-Self-administered interview questionnaire this tool consisted of 2 parts:

Part 1: include the nurse's demographic data such as age, educational level, educational qualification, years of experience.

Part 2: to assess maternity nurse knowledge regarding cardiac arrest during pregnancy and cardiopulmonary resuscitation. It includes 16 different questions. Each questions given a correct answer, the total number of correct answers were 59 answers, answer was scored as (2) for correct answer and (1) for incorrect answer. The total knowledge score was calculated by summation of the scores for the correct answers. The total possible score ranged from (1 to 118 marks) and means and standard deviations were calculated. The higher scores reflect higher levels of knowledge about CPR during pregnancy.

The total knowledge score was indicated as the following:

Total knowledge score was calculated as:

Adequate knowledge $\geq 60 \%$ of total knowledge score ranged from (71-118 marks).

Inadequate knowledge $<60 \%$ of total knowledge score ranged from (1-70 marks)

Tool II-maternity nurse practice observational checklist

It was constructed by the researcher after reviewing relevant literature. It was adopted from (Hui et a, 2011 et al., 2011, Rodrigues et al., 2014), and (Adams et al., 2016). It was concerned with assessing the maternity nurse practice regarding cardiopulmonary resuscitation during pregnancy. This part consisted of (66 steps) items that identify the steps of different cardiopulmonary resuscitation. Preparatory and basic intervention (12 steps), compression related practice (8steps), open air way and breathing technique include (15 steps), defibrillation technique (17 steps), and intubation and post resuscitation care related practice included (14 steps). Each step was scored as (1) for not done, and (2) for done, total practice score as satisfactory practice $\geq 75 \%$ of total practice score (99-132marks), unsatisfactory practice $<75 \%$ (1-99 marks).

\subsection{Pilot Study}

A pilot study was conducted on (10\%) of the studied nurses to test the feasibility, clarity and applicability of the developed tools, based on the finding of the pilot study.

\subsection{Tool Validity}

Tools developed by the researchers were examined by a panel of five experts (three cardiologists and two professors of maternity nursing speciality) to determine whether the included items clearly and adequately cover the domain of content addressed. The percentage of consensus among experts regarding the structured interviewing questionnaire was $94 \%$ and the pre-post-test was $87 \%$. 


\subsubsection{Tool Reliability}

Test-retest was repeated to the same sample of maternity nurses on two occasions and then compares the scores The Cronbach's coefficient alpha was 0.879 .

\subsection{Simulation Based Intervention}

The simulation intervention provided by the researchers using different strategies, and training sessions in individual basis, small and large group discussion strategies also were utilized. Simulation training was conducted at the woman's health nursing department training lab affiliated at faculty of nursing at Benha University. Researchers prepare the training lab with essential needed supplies as Gaumard's NOELLE maternal birthing simulator with a pregnant uterus was used for all simulations to enable the performance of breathing, chest compression, intubation. And defibrillation in addition a digital monitor displaying dynamic vital signs was readily to evaluate woman's condition and assessment fetal wellbeing device.

\subsubsection{Ethical Consideration}

An Informed consent was obtained from each maternity nurse at the first session. Each maternity nurse was informed that the participation in the study was voluntary and can withdraw at any time.

\subsubsection{Procedure}

The study was begun at the 1st of February 2015 after receiving the approval from the dean of faculty of nursing and the director of Benha university hospital to the end of July 2015. Maternity nurses were divided into 10 different small groups in order to avoid contradiction with their work, each group contain 5 to 6 nurses. Simulation intervention was implemented through 3 different sessions (one theoretical session and two practical training sessions) for each group the researchers provide firstly a session regarding theoretical knowledge about cardiac arrest and cardiopulmonary resuscitation during pregnancy (meaning, causes, factors aggravate cardiac arrest during pregnancy) at the practical sessions were concerned with training nurses regarding technique of cardiopulmonary resuscitation during pregnancy including breathing technique, chest compression technique, defibrillation and intubation technique.

\subsubsection{Evaluations}

The pre-posttest format was used in two times of assessment; the first was before the intervention, the second time was after the implementation of the simulation intervention which extends to three months. For evaluating practical skills maternity nurses were asked to demonstrate the procedure on the studied simulator for three different times and average of different score was calculated.

\subsection{Statistical Design}

Data analysis was performed using IBM SPSS (Statistical Package for Social Sciences) statistical software version 22. The data were explored. Descriptive statistics with mean and standard deviation (SD) for continuous variables and frequency for categorical variables were analyzed. Qualitative variables were compared using qui square test (X2) as the test of significance, paired and independent (t) test and ANOVA test was used to compare mean score between two and more groups respectively. Correlation coefficient (r) was used to evaluate association between studied variables. The p-value is the degree of significant. A significant level value was considered when $p$-value $\leq 0.05$ and a highly significant level value was considered when $p$-value $\leq 0.001$, while p-value $>0.05$ indicates non-significant results.

\section{Results}

Table 1: Reveals that more than half of the studied maternity nurses were aged (20-29) years, more than half $(54.0 \%)$ of them had a secondary nursing education. Moreover $38.0 \%$ of them had less than five years of experience. In addition more than half live in urban area. It also indicates that the highest percentage $78 \%$ previous training regarding CPR.

Table 2: Shows that, there was a highly statistically significant difference between mean scores of knowledge related to cardiac arrest during pregnancy between the pre and post intervention phases $(p<0.001)$.

Figure 1: Percentage distribution of total knowledge score of the studied nurses at different phases of the program.

Table 3: Reveals that, there was a highly statistically significance difference between total knowledge scores of maternity nurses and their educational qualification, years of experience and previous training regarding CPR. 
Table 4: Indicates that, there was a highly statistically significant difference between studied nurses' practice about basic intervention for cardiopulmonary resuscitation procedure between the pre and post intervention phases $(\mathrm{p}<0.001)$.

Table 5: Indicates that, there was a highly statistically significant difference between studied nurses 'practice related to applying chest compressions between the pre and post intervention phases $(p<0.001)$.

Table 6: Indicates that, there was a highly statistically significant difference between studied nurses' practice related to open airway and delivering breath technique at both the pre and post intervention phases $(\mathrm{p}<0.001)$.

Table 7: Shows that, there was a highly statistically significant difference between studied nurses' practice regarding defibrillator technique between the pre and post intervention phases $(\mathrm{p}<0.001)$.

Table 8: Indicates that, there was a highly statistically significant difference between studied nurses' practice scores related to applying intubation between the pre and post intervention phases $(\mathrm{p}<0.001)$.

Figure 2: Percentage distribution of total practice score of the studied nurses at different phases of the program.

Table 9: Reveals that, there was a highly statistically significance difference between total practice scores of maternity nurses and their previous training regarding CPR, at post intervention phase $(p<0.001)$ and, moreover there was statistically significance difference between total practice scores in relation to their educational qualification at post intervention phase $\left(<0.05^{*}\right)$.

Table 10: Shows that, that there was a highly positive association between studied nurses knowledge and practice scores at post intervention phase.

Table 1. Distribution of personnel characteristics of the studied nurses

\begin{tabular}{|c|c|c|}
\hline Variable & Frequency & $\%$ \\
\hline \multicolumn{3}{|l|}{ Age in years } \\
\hline $20-29$ & 26 & 52.0 \\
\hline $30-39$ & 20 & 40.0 \\
\hline $40-50$ & 4 & 8.0 \\
\hline Mean \pm SD & \multicolumn{2}{|c|}{$28.23 \pm 5.02$} \\
\hline \multicolumn{3}{|l|}{ Educational qualification } \\
\hline Secondary nursing education & 27 & 54.0 \\
\hline Technical nursing education & 18 & 36.0 \\
\hline Baculare of nursing & 5 & 10.0 \\
\hline \multicolumn{3}{|l|}{ Years of experience } \\
\hline $5-9$ & 19 & 38.0 \\
\hline $10-14$ & 8 & 16.0 \\
\hline $15-19$ & 11 & 22.0 \\
\hline $19-25$ & 12 & 24.0 \\
\hline Mean \pm SD & \multicolumn{2}{|c|}{$10.58 \pm 6.35$} \\
\hline \multicolumn{3}{|l|}{ Residence } \\
\hline Urban & 32 & 64.0 \\
\hline Rural & 18 & 36.0 \\
\hline \multicolumn{3}{|l|}{ Previous training regarding $\mathrm{CPR}$} \\
\hline No & 39 & 78 \\
\hline Yes & 11 & 22 \\
\hline
\end{tabular}


Table 2. Distribution of knowledge regarding cardiac arrest during pregnancy of the studied nurses at different phases of the intervention

\begin{tabular}{|c|c|c|c|c|c|}
\hline Knowledge & $\begin{array}{l}\text { No of } \\
\text { answers }\end{array}$ & $\begin{array}{c}\text { Pre-intervention } \\
\text { Mean } \pm \text { SD }\end{array}$ & $\begin{array}{c}\text { Post-intervention } \\
\text { Mean } \pm \text { SD }\end{array}$ & $\begin{array}{c}\text { Paired t } \\
\text { test }\end{array}$ & P value \\
\hline Definition of cardiac pulmonary arrest & 2 & $1.3200 \pm .47121$ & $1.7600 \pm .43142$ & -4.831 & $<0.001 * *$ \\
\hline Causes of cardiac pulmonary arrest in pregnancy & 6 & $1.7800 \pm 1.20017$ & $4.9000 \pm 1.75255$ & -10.995 & $<0.001 * *$ \\
\hline Complication of cardiac arrest during pregnancy & 5 & $1.7400 \pm .80331$ & $3.7000 \pm 1.47427$ & -8.031 & $<0.001 * *$ \\
\hline Management of cardio pulmonary during pregnancy & 5 & $1.5600 \pm .86094$ & $4.1800 \pm 1.40973$ & -9.837 & $<0.001 * *$ \\
\hline \multicolumn{6}{|l|}{ Cardiopulmonary resuscitation : } \\
\hline Definition of CPR & 2 & $1.2400 \pm .43142$ & $1.8400 \pm .37033$ & -7.425 & $<0.001 * *$ \\
\hline Importance of CPR & 6 & $1.9800 \pm .76904$ & $4.9800 \pm 1.65973$ & -11.813 & $<0.001 * *$ \\
\hline General principles of CPR & 4 & $1.6400 \pm .48487$ & $3.3600 \pm .96384$ & -11.369 & $<0.001 * *$ \\
\hline Indication for CPR & 3 & $1.3800 \pm .49031$ & $2.8400 \pm .42185$ & -15.992 & $<0.001 * *$ \\
\hline Ways to open the airway & 3 & $1.2600 \pm .44309$ & $2.6200 \pm .66670$ & -11.627 & $<0.001 * *$ \\
\hline Depth of chest compression & 3 & $1.3200 \pm .5107$ & $2.8200 \pm .43753$ & -13.908 & $<0.001 * *$ \\
\hline Rate of compressions & 2 & $1.2800 \pm .45356$ & $1.6600 \pm .47852$ & -4.030 & $<0.001 * *$ \\
\hline Rate of chest compression to the breathing & 2 & $1.3000 \pm .46291$ & $1.9400 \pm .23990$ & -8.041 & $<0.001 * *$ \\
\hline Site at which starts chest compressions & 2 & $1.3400 \pm .47852$ & $1.7800 \pm .41845$ & -4.831 & $<0.001 * *$ \\
\hline Complications of CPR & 5 & $1.7400 \pm .82833$ & $4.5600 \pm 1.10951$ & -15.667 & $<0.001 * *$ \\
\hline $\begin{array}{l}\text { Physiological changes during pregnancy affect cardio pulmonary } \\
\text { resuscitation. }\end{array}$ & 5 & $1.4000 \pm .69985$ & $4.5000 \pm 1.16496$ & -14.467 & $<0.001 * *$ \\
\hline $\begin{array}{l}\text { Drugs used during cardio pulmonary resuscitation during } \\
\text { pregnancy }\end{array}$ & 4 & $1.3200 \pm .47121$ & $3.7400 \pm .63278$ & -21.116 & $<0.001 * *$ \\
\hline Total & 59 & $23.6000 \pm 4.00510$ & $51.1800 \pm 3.91590$ & -35.411 & $<0.001 * *$ \\
\hline
\end{tabular}

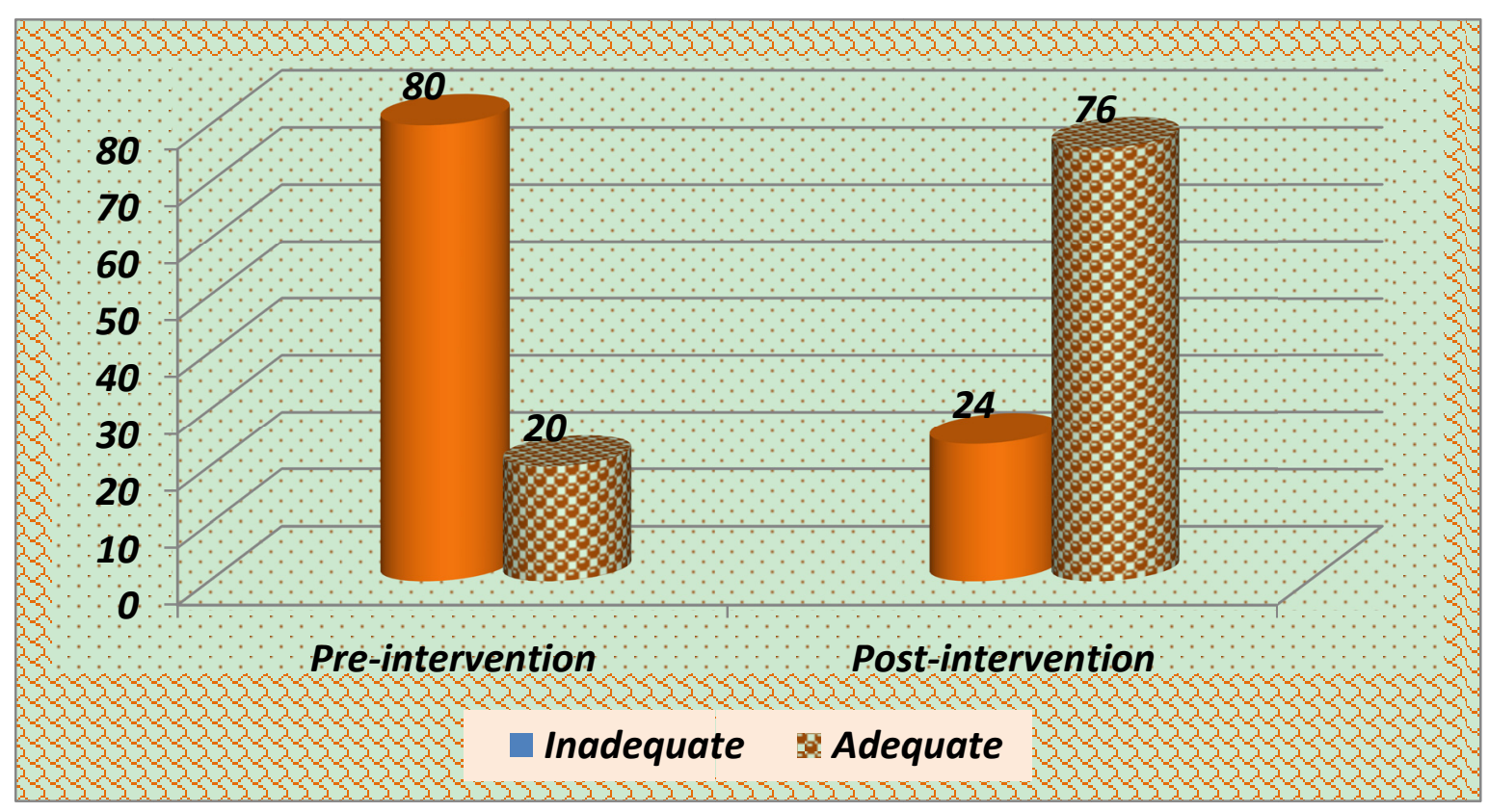

Figure 1. Percentage distribution of total knowledge score of the studied nurses at different phases of the program 
Table 3. Relation between studied nurses' total knowledge score and their personnel characteristics at different phases of intervention

\begin{tabular}{|c|c|c|c|c|c|c|c|c|}
\hline \multirow[b]{2}{*}{ Variable } & \multicolumn{4}{|c|}{ Pre-intervention } & \multicolumn{4}{|c|}{ Post-intervention } \\
\hline & Mean \pm SD & F test & $\begin{array}{c}\text { Independe } \\
\text { nt t test }\end{array}$ & Pvalue & Mean \pm SD & F test & $\begin{array}{c}\text { Independe } \\
\text { nt } t \text { test }\end{array}$ & Pvalue \\
\hline 20- & $22.9231 \pm 4.61236$ & & & & $51.1923 \pm 4.08920$ & & & \\
\hline $30-$ & $23.9500 \pm 3.06894$ & & & & $50.7500 \pm 3.87808$ & & & \\
\hline$\geq 40$ & $26.2500 \pm 3.30404$ & & & & $53.2500 \pm 2.98608$ & & & \\
\hline Technical nursing education & $22.7222 \pm 4.34952$ & & & & $50.2222 \pm 4.09527$ & & & \\
\hline Baculare of nursing & $30.2000 \pm .44721$ & & & & $57.2000 \pm .44721$ & & & \\
\hline Years of experience & & 39.72 & & $<0.001 * *$ & & 38.14 & & $<0.001 * *$ \\
\hline $5-9$ & $20.2609 \pm 2.54447$ & & & & $47.9130 \pm 2.77837$ & & & \\
\hline $10-14$ & $23.0000 \pm .81650$ & & & & $50.7500 \pm .50000$ & & & \\
\hline Rural & $22.9688 \pm 3.30673$ & & & & $51.0938 \pm 4.36525$ & & & \\
\hline Urban & $24.7222 \pm 4.92061$ & & & & $51.3333 \pm 3.06786$ & & & \\
\hline Previous training regarding & & & 3.09 & $<0.001 * *$ & & 2.88 & & $<0.001^{* *}$ \\
\hline \multicolumn{9}{|l|}{ CPR } \\
\hline No & $22.7949 \pm 3.83323$ & & & & $50.4872 \pm 3.89920$ & & & \\
\hline Yes & $26.4545 \pm 3.35749$ & & & & $53.6364 \pm 2.97566$ & & & \\
\hline
\end{tabular}

Table 4. Distribution of practice regarding cardiopulmonary resuscitation procedure among the studied nurses at different phases of intervention

\begin{tabular}{|c|c|c|c|c|c|c|c|c|c|c|}
\hline \multirow[t]{3}{*}{ Basic intervention } & \multicolumn{4}{|c|}{ Pre-intervention } & \multicolumn{4}{|c|}{ Post-intervention } & \multirow{3}{*}{ Chi-square $\chi^{2}$} & \multirow{3}{*}{$\begin{array}{c}\mathbf{P} \\
\text { Value }\end{array}$} \\
\hline & \multicolumn{2}{|c|}{ Not Done } & \multicolumn{2}{|c|}{ Done } & \multicolumn{2}{|c|}{ Not Done } & \multicolumn{2}{|c|}{ Done } & & \\
\hline & No & $\%$ & No & $\%$ & No & $\%$ & No & $\%$ & & \\
\hline \multicolumn{11}{|l|}{ Pre procedure: } \\
\hline Activate maternal cardiac arrest team & 45 & 90.0 & 5 & 10.0 & 6 & 12.0 & 44 & 88.0 & 60.86 & $<0.001^{* *}$ \\
\hline $\begin{array}{l}\text { Document time of onset of maternal } \\
\text { cardiac arrest }\end{array}$ & 44 & 88.0 & 6 & $12.0 \%$ & 6 & 12.0 & 44 & 88.0 & 57.76 & $<0.001 * *$ \\
\hline Keep women at left lateral position & 43 & 86.0 & 7 & 14.0 & 9 & 18.0 & 41 & 82.0 & 46.31 & $<0.001 * *$ \\
\hline Estimate gestational age & 43 & 86.0 & 7 & 14.0 & 7 & 14.0 & 43 & 86.0 & 51.84 & $<0.001 * *$ \\
\hline Prepare equipment & 46 & 92.0 & 4 & 8.0 & 7 & 14.0 & 43 & 86.0 & 61.06 & $<0.001 * *$ \\
\hline Keep privacy & 39 & 78.0 & 11 & 22.0 & 7 & 14.0 & 43 & 86.0 & 41.22 & $<0.001 * *$ \\
\hline \multicolumn{11}{|l|}{ During procedure (Basic intervention): } \\
\hline Assess woman's consciousness & 41 & 82.0 & 9 & 18.0 & 6 & 12.0 & 44 & 88.0 & 49.17 & $<0.001 * *$ \\
\hline Don't transport women & 41 & 82.0 & 9 & 18.0 & 9 & 18.0 & 41 & 82.0 & 40.96 & $<0.001 * *$ \\
\hline Immediately call out for help & 43 & 86.0 & 7 & 14.0 & 6 & 12.0 & 44 & 88.0 & 54.78 & $<0.001 * *$ \\
\hline Palpate the carotid artery & 43 & 86.0 & 7 & 14.0 & 7 & 14.0 & 43 & 86.0 & 51.81 & $<0.001 * *$ \\
\hline Ensure a clear air way & 45 & 90.0 & 5 & 10.0 & 6 & 12.0 & 44 & 88.0 & 60.86 & $<0.001 * *$ \\
\hline Assess fetal heart sound & 41 & 82.0 & 9 & 18.0 & 6 & 12.0 & 44 & 88.0 & 49.17 & $<0.001^{* *}$ \\
\hline
\end{tabular}


Table 5. Distribution of practice about demonstration of chest compression technique among the studied nurses at different phases of the program

\begin{tabular}{|c|c|c|c|c|c|c|c|c|c|c|}
\hline \multirow[t]{3}{*}{ applying Chest compressions } & \multicolumn{4}{|c|}{ Pre-intervention } & \multicolumn{4}{|c|}{ Post-intervention } & \multirow{3}{*}{$\begin{array}{l}\text { Chi-squ } \\
\text { are } \chi^{2}\end{array}$} & \multirow{3}{*}{$\begin{array}{c}\mathbf{P} \\
\text { Value }\end{array}$} \\
\hline & \multicolumn{2}{|c|}{ Not Done } & \multicolumn{2}{|c|}{ Done } & \multicolumn{2}{|c|}{ Not Done } & \multicolumn{2}{|c|}{ Done } & & \\
\hline & No & $\%$ & No & $\%$ & No & $\%$ & No & $\%$ & & \\
\hline Hand position & 42 & 84.0 & 8 & $16.0 \%$ & 6 & 12.0 & 44 & 88.0 & 51.92 & $<0.001^{* *}$ \\
\hline $\begin{array}{l}\text { Place women at supine position during } \\
\text { compression }\end{array}$ & 44 & 88.0 & 6 & 12.0 & 3 & 6.0 & 47 & 94.0 & 67.48 & $<0.001^{* *}$ \\
\hline $\begin{array}{l}\text { Place hand in the centre of chest, slightly above } \\
\text { the sternum }\end{array}$ & 39 & 78.0 & 11 & 22.0 & 6 & 12.0 & 44 & 88.0 & 44.00 & $<0.001 * *$ \\
\hline $\begin{array}{l}\text { Chest compressions should be performed at a } \\
\text { rate of at least } 100 \text { per minute. }\end{array}$ & 46 & 92.0 & 4 & 8.0 & 9 & 18.0 & 41 & 82.0 & 55.31 & $<0.001 * *$ \\
\hline $\begin{array}{l}\text { Chest compressions should be performed at a } \\
\text { depth of at least } 2 \text { inch }(5 \mathrm{~cm})\end{array}$ & 40 & 80.0 & 10 & 20.0 & 6 & 12.0 & 44 & 88.0 & 46.53 & $<0.001 * *$ \\
\hline $\begin{array}{l}\text { Allow complete chest recoil after each chest } \\
\text { compression }\end{array}$ & 40 & 80.0 & 10 & 20.0 & 12 & 24.0 & 38 & 76.0 & 31.41 & $<0.001 * *$ \\
\hline Minimize interruption & 38 & 76.0 & 12 & 24.0 & 8 & 16.0 & 42 & 84.0 & 36.23 & $<0.001 * *$ \\
\hline $\begin{array}{l}\text { Perfume continuous manual left uterine } \\
\text { displacement }\end{array}$ & 41 & 82.0 & 9 & 18.0 & 11 & 22.0 & 39 & 78.0 & 36.05 & $<0.001 * *$ \\
\hline
\end{tabular}

Table 6. Distribution of practice of open air way and delivering breath technique among the studied nurses at different phases of the intervention

\begin{tabular}{|c|c|c|c|c|c|c|c|c|c|c|}
\hline \multirow[t]{3}{*}{ Delivering breath } & \multicolumn{4}{|c|}{ Pre-intervention } & \multicolumn{4}{|c|}{ Post-intervention } & \multirow{3}{*}{$\begin{array}{c}\text { Chi-square } \\
\chi^{2}\end{array}$} & \multirow{3}{*}{$\begin{array}{c}P \\
\text { Value }\end{array}$} \\
\hline & \multicolumn{2}{|c|}{ Not Done } & \multicolumn{2}{|c|}{ Done } & \multicolumn{2}{|c|}{ Not Done } & \multicolumn{2}{|c|}{ Done } & & \\
\hline & No & $\%$ & No & $\%$ & No & $\%$ & No & $\%$ & & \\
\hline \multicolumn{11}{|l|}{ Open the airway and give breathing: } \\
\hline \multicolumn{11}{|l|}{ Head-tilt/chin-lift manoeuvre } \\
\hline Hand position & 44 & 88.0 & 6 & 12.0 & 10 & 20.0 & 40 & 80.0 & 46.53 & $<0.001 * *$ \\
\hline Place the fingers of the other hand under the bony part & 42 & 84.0 & 8 & 16.0 & 9 & 18.0 & 41 & 82.0 & 43.57 & $<0.001 * *$ \\
\hline $\begin{array}{l}\text { Grasp the angles of the woman 's lower jaw and lifting } \\
\text { with both hands }\end{array}$ & 46 & 92.0 & 4 & 8.0 & 7 & 14.0 & 43 & 86.0 & 61.06 & $<0.001^{* *}$ \\
\hline Ensure a clear air way & 43 & 86.0 & 7 & 14.0 & 6 & 12.0 & 44 & 88.0 & 54.78 & $<0.001 * *$ \\
\hline \multicolumn{11}{|l|}{ Deliver breathing } \\
\hline $\begin{array}{l}\text { A- perform the bag valve mask } \\
\text { Ensure a tight seal between the mask and the woman's face }\end{array}$ & 46 & 92.0 & 4 & 8.0 & 6 & 12.0 & 44 & 88.0 & 64.10 & $<0.001^{* *}$ \\
\hline Squeeze the bag with two hand & 46 & 92.0 & 4 & 8.0 & 7 & 14.0 & 43 & 86.0 & 61.06 & $<0.001 * *$ \\
\hline Administer $100 \%$ oxygen at 15 litre/minute & 38 & 76.0 & 12 & 24.0 & 11 & 22.0 & 39 & 78.0 & 29.17 & $<0.001^{* *}$ \\
\hline Compress the ambo-bag in rhythmical fashion & 39 & 78.0 & 11 & 22.0 & 9 & 18.0 & 41 & 82.0 & 35.06 & $<0.001 * *$ \\
\hline Attach the bag to an oxygen source & 38 & 76.0 & 12 & 24.0 & 10 & 20.0 & 40 & 80.0 & 31.41 & $<0.001^{* *}$ \\
\hline Pinch the woman's nostrils closed & 40 & 80.0 & 10 & 20.0 & 7 & 14.0 & 43 & 86.0 & 43.71 & $<0.001 * *$ \\
\hline Put the mouth completely over the woman's mouth & 44 & 88.0 & 6 & 12.0 & 7 & 14.0 & 43 & 86.0 & 54.78 & $<0.001 * *$ \\
\hline After 30 chest compression, give 2 breaths & 45 & 90.0 & 5 & 10.0 & 9 & 18.0 & 41 & 82.0 & 52.17 & $<0.001^{* *}$ \\
\hline $\begin{array}{l}\text { Deliver each rescue breath over one second with enough } \\
\text { force }\end{array}$ & 47 & 94.0 & 3 & 6.0 & 10 & 20.0 & 40 & 80.0 & 55.85 & $<0.001 * *$ \\
\hline Give sufficient tidal volume to produce visible chest rise & 45 & 90.0 & 5 & 10.0 & 6 & 12.0 & 44 & 88.0 & 60.86 & $<0.001^{* *}$ \\
\hline Avoid excessive ventilation & 47 & 94.0 & 3 & 6.0 & 12 & 24.0 & 38 & 76.0 & 50.64 & $<0.001 * *$ \\
\hline
\end{tabular}


Table 7. Distribution of practice of defibrillator technique among the studied at different phases of the intervention

\begin{tabular}{|c|c|c|c|c|c|c|c|c|c|c|}
\hline \multirow[t]{3}{*}{ Basic intervention } & \multicolumn{4}{|c|}{ Pre-intervention } & \multicolumn{4}{|c|}{ Post-intervention } & \multirow{3}{*}{$\begin{array}{c}\text { Chi- } \\
\text { square } \\
\chi^{2}\end{array}$} & \multirow{3}{*}{$\begin{array}{c}\mathbf{P} \\
\text { Value }\end{array}$} \\
\hline & \multicolumn{2}{|c|}{ Not Done } & \multicolumn{2}{|c|}{ Done } & \multicolumn{2}{|c|}{ Not Done } & \multicolumn{2}{|c|}{ Done } & & \\
\hline & No & $\%$ & No & $\%$ & No & $\%$ & No & $\%$ & & \\
\hline Verify that woman is unresponsive. & 42 & 84.0 & 8 & 16.0 & 10 & 20.0 & 40 & 80.0 & 41.02 & $<0.001 * *$ \\
\hline $\begin{array}{l}\text { Verify ECG reading of ventricular tachycardia, or } \\
\text { ventricular fibrillation }\end{array}$ & 40 & 80.0 & 10 & 20.0 & 10 & 20.0 & 40 & 80.0 & 36.00 & $<0.001^{* *}$ \\
\hline Plug defibrillator into electric outlet. & 39 & 78.0 & 11 & 22.0 & 13 & 26.0 & 37 & 74.0 & 27.08 & $<0.001^{* *}$ \\
\hline Turn on defibrillator power. & 41 & 82.0 & 9 & 18.0 & 9 & 18.0 & 41 & 82.0 & 40.96 & $<0.001^{* *}$ \\
\hline $\begin{array}{l}\text { Prepare woman and/or paddles with proper conductive } \\
\text { agent }\end{array}$ & 37 & 74.0 & 13 & 26.0 & 10 & 20.0 & 40 & 80.0 & 29.26 & $<0.001^{* *}$ \\
\hline Checks that defibrillator is in asynchronous mode. & 43 & 86.0 & 7 & 14.0 & 9 & 18.0 & 41 & 82.0 & 46.31 & $<0.001^{* *}$ \\
\hline Deliver defibrillated & & & & & & & & & & \\
\hline Turn on ECG recorder. & 42 & 84.0 & 8 & 16.0 & 9 & 18.0 & 41 & 82.0 & 43.57 & $<0.001 * *$ \\
\hline Command all persons to move away from bed & 40 & 80.0 & 10 & 20.0 & 8 & 16.0 & 42 & 84.0 & 41.02 & $<0.001^{* *}$ \\
\hline Turn off oxygen source during actual & 40 & 80.0 & 10 & 20.0 & 10 & 20.0 & 40 & 80.0 & 36.00 & $<0.001 * *$ \\
\hline Stand away from bed area & 41 & 82.0 & 9 & 18.0 & 11 & 22.0 & 39 & 78.0 & 36.05 & $<0.001 * *$ \\
\hline Places the paddles at proper site on chest. & 38 & 76.0 & 12 & 24.0 & 10 & 20.0 & 40 & 80.0 & 31.41 & $<0.001 * *$ \\
\hline Apply paddles with firm pressure(11kg pressure) & 37 & 74.0 & 13 & 26.0 & 10 & 20.0 & 40 & 80.0 & 29.26 & $<0.001 * *$ \\
\hline Depress discharge buttons on defibrillator. & 42 & 84.0 & 8 & 16.0 & 12 & 24.0 & 38 & 76.0 & 36.23 & $<0.001 * *$ \\
\hline $\begin{array}{l}\text { Deliver } 3 \text { stacked shocks, with biphasic shock energy of } \\
120 \text { to } 200\end{array}$ & 38 & 76.0 & 12 & 24.0 & 13 & 26.0 & 37 & 74.0 & 25.01 & $<0.001 * *$ \\
\hline $\begin{array}{l}\text { Compressions should be resumed immediately delivery } \\
\text { of the electric shock }\end{array}$ & 36 & 72.0 & 14 & 28.0 & 13 & 26.0 & 37 & 74.0 & 21.16 & $<0.001^{* *}$ \\
\hline Assess women cardiac rhythm & 35 & 70.0 & 15 & 30.0 & 10 & 20.0 & 40 & 80.0 & 25.25 & $<0.001 * *$ \\
\hline $\begin{array}{l}\text { If first defibrillation unsuccessful, immediately charge } \\
\text { paddles to } 300 \text { joules }\end{array}$ & 33 & 66.0 & 17 & 34.0 & 15 & 30.0 & 35 & 70.0 & 12.98 & $<0.001 * *$ \\
\hline
\end{tabular}

Table 8. Distribution of practice of intubation technique among the studied nurses at different phases of intervention

\begin{tabular}{|c|c|c|c|c|c|c|c|c|c|c|}
\hline \multirow[t]{3}{*}{ Application of intubation } & \multicolumn{4}{|c|}{ Pre-intervention } & \multicolumn{4}{|c|}{ Post-intervention } & \multirow{3}{*}{$\begin{array}{c}\text { Chi-square } \\
\chi^{2}\end{array}$} & \multirow{3}{*}{$\begin{array}{c}P \\
\text { Value }\end{array}$} \\
\hline & \multicolumn{2}{|c|}{ Not Done } & \multicolumn{2}{|c|}{ Done } & \multicolumn{2}{|c|}{ Not Done } & \multicolumn{2}{|c|}{ Done } & & \\
\hline & No & $\%$ & No & $\%$ & No & $\%$ & No & $\%$ & & \\
\hline $\begin{array}{l}\text { Applying intubation: } \\
\text { Continue to ventilation \& oxygenation }\end{array}$ & 40 & 80.0 & 10 & 20.0 & 7 & 14.0 & 43 & 86.0 & 43.71 & $<0.001 * *$ \\
\hline Prepare Equipment & 47 & 94.0 & 3 & 6.0 & 6 & 12.0 & 44 & 88.0 & 67.48 & $<0.001 * *$ \\
\hline The anaesthetist may require & 46 & 92.0 & 4 & 8.0 & 6 & 12.0 & 44 & 88.0 & 64.10 & $<0.001 * *$ \\
\hline $\begin{array}{l}\text { Recommence ventilation and oxygenation } \\
\text { once intubation is completed }\end{array}$ & 42 & 84.0 & 8 & 16.0 & 5 & 10.0 & 45 & 90.0 & 54.95 & $<0.001 * *$ \\
\hline Continues CPR once intubation & 43 & 86.0 & 7 & 14.0 & 8 & 16.0 & 42 & 84.0 & 49.02 & $<0.001 * *$ \\
\hline Established venous access & 44 & 88.0 & 6 & 12.0 & 8 & 16.0 & 42 & 84.0 & 51.92 & $<0.001 * *$ \\
\hline Asepsis should be maintaining & 43 & 86.0 & 7 & 14.0 & 7 & 14.0 & 43 & 86.0 & 51.84 & $<0.001 * *$ \\
\hline The correct rate of infusion is required & 46 & 92.0 & 4 & 8.0 & 7 & 14.0 & 43 & 86.0 & 61.06 & $<0.001^{* *}$ \\
\hline Accurate documentation & 45 & 90.0 & 5 & 10.0 & 7 & 14.0 & 43 & 86.0 & 57.85 & $<0.001^{* *}$ \\
\hline \multicolumn{11}{|l|}{ Applying Post resuscitation care: } \\
\hline Assess woman 's condition & 45 & 90.0 & 5 & 10.0 & 8 & $16.0 \%$ & 42 & 84.0 & 54.95 & $<0.001 * *$ \\
\hline Check arterial blood gases & 43 & 86.0 & 7 & 14.0 & 8 & $16.0 \%$ & 42 & 84.0 & $49 . .02$ & $<0.001 * *$ \\
\hline Monitor woman's cardiac rhythm & 39 & 78.0 & 11 & 22.0 & 9 & $18.0 \%$ & 41 & 82.0 & 36.05 & $<0.001 * *$ \\
\hline Continue respiratory therapy & 36 & 72.0 & 14 & 28.0 & 7 & $14.0 \%$ & 43 & 86.0 & 34.31 & $<0.001 * *$ \\
\hline Assess woman's level of consciousness & 38 & 76.0 & 12 & 24.0 & 5 & $10.0 \%$ & 45 & 90.0 & 44.43 & $<0.001 * *$ \\
\hline
\end{tabular}




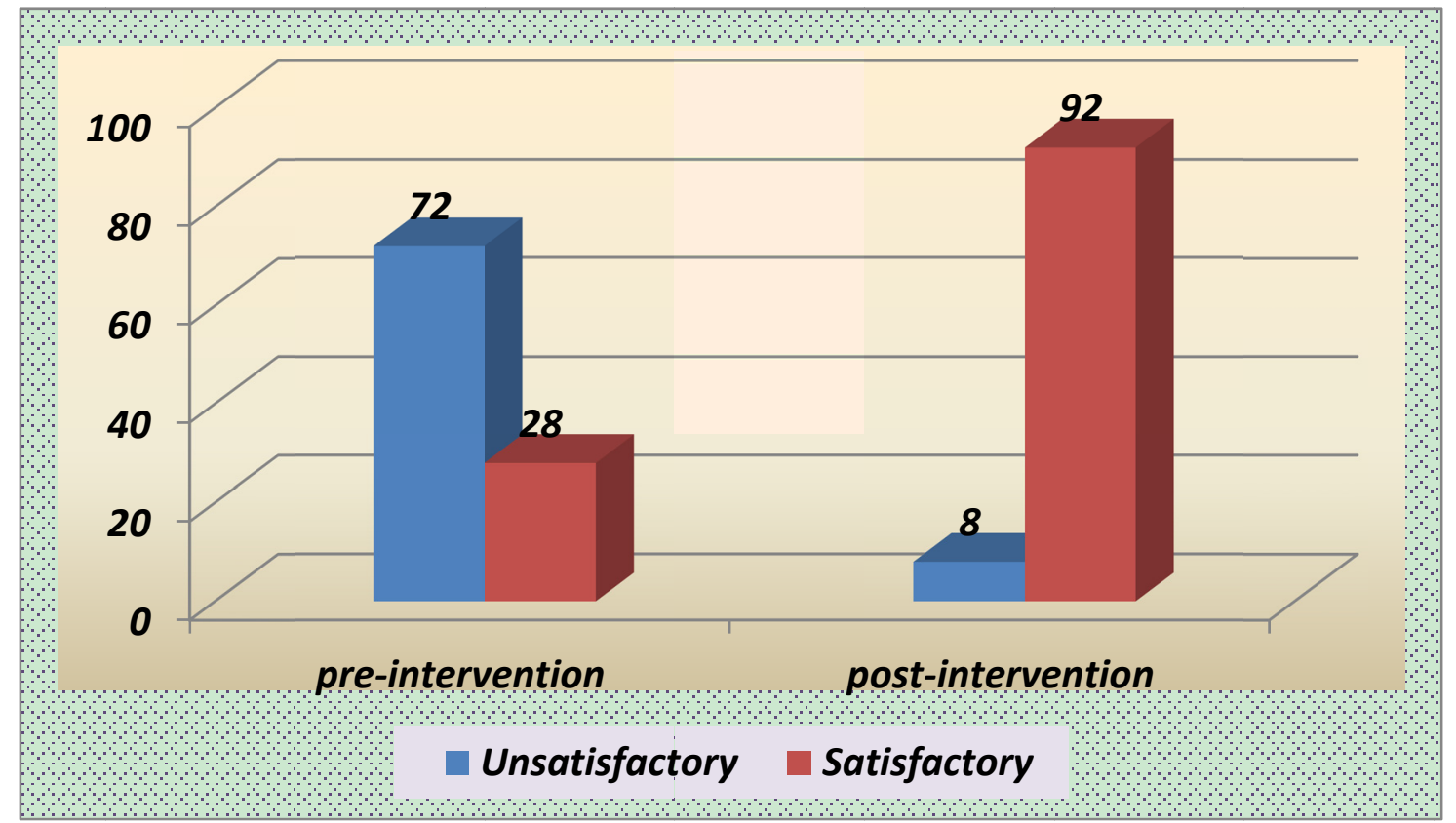

Figure 2. Percentage distribution of total practice score of the studied nurses regarding CPR during pregnancy at different phases of intervention

Table 9. Relation between studied nurses total practice score and their personnel characteristics at different phases of intervention

\begin{tabular}{|c|c|c|c|c|c|c|c|c|}
\hline \multirow[b]{2}{*}{ Variable } & \multicolumn{4}{|c|}{ Pre-intervention } & \multicolumn{4}{|c|}{ Post-intervention } \\
\hline & Mean \pm SD & F test & $\begin{array}{c}\text { Independent } t \\
\text { test }\end{array}$ & P value & Mean \pm SD & F test & & P value \\
\hline Age in years & & 0.530 & & $>0.05$ & & 1.72 & & $>0.05$ \\
\hline 20- & $73.8462 \pm 11.73607$ & & & & $118.8462 \pm 9.455$ & & & \\
\hline $30-$ & $77.7500 \pm 15.36529$ & & & & $120.9500 \pm 8.331$ & & & \\
\hline $40-52$ & $77.7500 \pm 12.97112$ & & & & $111.7500 \pm 10.59$ & & & \\
\hline Technical nursing education & $76.7778 \pm 7.53467$ & & & & $123.3333 \pm 5.434$ & & & \\
\hline Baculare of nursing & $107.2000 \pm 14.5155$ & & & & $130.0000 \pm .0000$ & & & \\
\hline Years of experience & & 27.57 & & $>0.05$ & & 0.258 & & $>0.05$ \\
\hline 5- & $70.2632 \pm 4.60485$ & & & & $116.4737 \pm 8.342$ & & & \\
\hline $10-$ & $67.2500 \pm 3.24037$ & & & & $110.1250 \pm 7.510$ & & & \\
\hline Rural & $76.0313 \pm 11.89872$ & & & & $119.6563 \pm 9.265$ & & & \\
\hline Urban & $75.1667 \pm 15.76761$ & & & & $118.1667 \pm 9.363$ & & & \\
\hline Previous training regarding & & 2.11 & & $>0.05$ & & & & $<0.001 * *$ \\
\hline CPR & & & & & & & 261 & \\
\hline No & $72.7692 \pm 8.65558$ & & & & $117.7692 \pm 9.609$ & & 2.01 & \\
\hline Yes & $86.1818 \pm 20.57580$ & & & & $123.9091 \pm 5.872$ & & & \\
\hline
\end{tabular}


Table 10. Correlation between studied nurses' knowledge and practice among studied nurses

\begin{tabular}{lcccc}
\hline & Variable & \multicolumn{2}{c}{ Practice pre } & \multicolumn{2}{c}{ Practice post } \\
& r & P value & r & P value \\
\hline Knowledge pre & -.186 & .195 & - & - \\
Knowledge post & -- & -- & $.485^{* *}$ & .000 \\
\hline
\end{tabular}

**. Correlation is significant at the 0.01 level (2-tailed).

\section{Discussion}

Nurses are usually the primary witnesses to in-hospital sudden cardiac arrests. Training of maternity nurses has an impact on the efficiency and outcome of cardiopulmonary resuscitation (CPR) during pregnancy. So, the aim of present study was to evaluate the effect of simulation based educational intervention concerning CPR during pregnancy among maternity nurses. The present study findings approved that the utilization of a simulation based educational intervention concerning cardiopulmonary resuscitation (CPR) during pregnancy increase the knowledge and skills of maternity nurses and motivated them to comply with training.

Regarding the socio-demographic characteristics of the maternity nurses, the present findings showed that studied nurses age ranged from 20-50 years, and about one half of studied maternity nurses had a age group ranged from 20 to 25 years with years of experience ranged from 5-9 with mean of (10.58 \pm 6.35$)$, and more than one half of them had a secondary nursing education. These study findings are agreed with Hussain et al. (2009) in the study at Bahrain, to evaluate nurses knowledge regarding cardiopulmonary resuscitation. It was reported that studied nurses' age ranged from 20-50 years and the majority had secondary nursing education, in addition, average years of experience was 12 years. Also (Mustafa \& Jaddoue) in the study at Al-Najaf city's teaching hospital to evaluate Knowledge of nurses towards cardiopulmonary resuscitation.

In addition, the result of the current study showed that the majority of nurses hadn't previous training course related CPR during pregnancy. This result is in accordance with Damjan et al. (2012). in the study to evaluate effects of using of the impedance threshold device in cardiopulmonary resuscitation. Who stated that more than third of the nurses don't have any previous training regarding CPR, and mostly all of them were willing to attend training program. On the other hand, Nagashema et al. (2012) in the study to investigate nurses' knowledge and experience in cardiopulmonary resuscitation, was in contrast with the present study and stated that the majority of the nurses are much interested in CPR, and most of them had received education and training in CPR as students or after the graduation.

Additionally, the finding of the present study proved majority of studied maternity nurses' had inadequate level of knowledge regarding all knowledge items concerning CPR during pregnancy at pre intervention phase. These findings may be due to that there is lack of implementing educational training program regarding cardiopulmonary resuscitation for pregnant women at the studied setting. These findings are agreed with (Cohen SE, et al., 2008) in the study to evaluate knowledge of health care provider regarding cardiopulmonary resuscitation of pregnant women. Who pointed out that health care providers knowledge regarding important of basic concepts, including the need for LUD and the potential benefit of early caesarean delivery during cardiac arrest, was inadequate. And added that advanced cardiac life support (ACLS) training is highly recommended.

Concerning effect of educational intervention on maternity nurses knowledge, the present study findings illustrated that was significantly improvement of nurses' knowledge immediately post intervention. These findings were in agreement with (Abd-Allah et al., 2017) in Egypt in the study to investigate effect of educational program on cardiopulmonary resuscitation on nursing student's performance. Who mentioned that educational program is positively improve knowledge of the studied nursing student. In addition the present study findings represented that there was significantly increase of total knowledge score post intervention.

As regard the practice of CPR during pregnancy among maternity nurses, the present study findings proved that the majority of the studied maternity nurses had un satisfactory practice regarding implementation of CPR during pregnancy at pre the program phase, which has been strongly increased immediately post intervention. This result was in agreement with (Elazazay et al., 2012). In Egypt in the study to determine the effect of cardiopulmonary resuscitation training program on nurses knowledge and practice at cancer institute, who mentioned that the nurses performance was very poor in all performance items at pre intervention phase, and their performance has been strongly improved immediately post intervention. These findings may be due to that the use of simulation gives the learner the opportunity to experience uncommon scenarios, receive feedback, and perhaps most importantly correct mistakes before they become part of the learner's. In addition simulation gives 
a chance for learners to apply procedure with any fear or distress.

In relation to the studied maternity nurse practice regarding application of chest compression technique, the study finding represented that, nurses had unsatisfactory practice at pre intervention phase and highly improved immediately post intervention. These finding was agreed with (Meissner, 2012). In the study to investigate basic life support skills among high school students before and after resuscitation training who observed significant improvement and good retention rate of CPR practice after program.

Moreover the present study findings showed that the total practice score of the studied nurses regarding CPR during pregnancy was unsatisfactory at pre intervention and become more satisfactory at post intervention, these finding was highly supported by (Elazazay, 2012) in the previous mentioned study, it was indicated that all nurses had unsatisfactory performance at pre the program (100\%), while $98.2 \%$ of them had good performance immediately post the program.

As regard the relation between the total knowledge score and personal characteristic, the present study findings represented that there were a statistically significant relation between nurses 'knowledge and educational qualifications post intervention, this result agrees with (Abd-Allah et al,. 2017). In The previous mentioned study, who found a statistically significant relation between level of education and knowledge. Moreover regarding the relation between knowledge and age, the present study proved that there was a no significant relation between knowledge pre, post the program and nurses' age. These finding are in accordance with (Parajulee 2011). in Nepal, in the study to investigate nurses knowledge regarding cardiopulmonary resuscitation in a tertiary care teaching hospital in Nepal, the study findings reported that there was no significant relation between the total knowledge score and age of the respondent, while (Aly, 2010) in Egypt in the study to evaluate impact of a basic life support training program at emergency room on nurses' knowledge and performance, contradicting this result and stated that there is significant relation between age and total basic life support knowledge scores.

As regarding relation between knowledge and years of experience, the finding showed there was no significant relation pre, post the program, this might be attributed that nursing education system is not preparing nurses to be effective in CPR especially and knowledge will be forgotten and deteriorated by time cone in the same line with (Parajulee et al., 2011). In the previous mentioned study, who added that there was no significant relation between the total knowledge score and the duration of experience. On the other hand, the finding was in disagreement with (Al Kandary et al., 2012) in the study to evaluate competence in cardio-pulmonary resuscitation among qualified nurses who proved significant relation between years of experience and knowledge scores.

On other hand there was a highly statically significant relation between total knowledge score of the studied nurses and previous training intervention at the post-intervention phase. This may be due to that training had a pivotal role in improving nurses' knowledge and practice and help nurses to be confident and decrease anxiety toward CPR. These findings agreed with (Kalhori, 2017). In the study to compare the cardiopulmonary resuscitation knowledge between hospital nurses and emergency medical personnel, it was pointed out that there was significantly higher in nurses who had passed basic CPR training courses compared with those who had not $(\mathrm{P}=0.018)$.

Furthermore, the results of the current study showed that that there were no statistically significant relations between nurses' practice and educational qualifications pre intervention while there was statically difference at post intervention also regarding the relation between the total practice score and previous training there was a highly statically significant post intervention.

The findings of the study may be related to the previous training which improves nurse's skills as regarding the correlation between total knowledge and practice scores of studied maternity nurse, the present study indicated that there was a highly positive association between total knowledge and practice scores at the post intervention phase, these findings agreed with (Aly, 2010) in the previous mentioned study who reported that there was significant correlation between total basic life support knowledge and practice scores.

\section{Conclusion}

Study concluded that simulation based intervention is highly improved maternity nurses knowledge, and competence regarding cardio pulmonary resuscitation during pregnancy.

\section{Recommendation}

Simulation based training regarding cardiopulmonary resuscitation should be provided for all obstetrics health care givers. 


\section{References}

Abd-Allah, E.A., Abd-Alla, K.F., \& Mahmoud, A.S. (2017). Effect of Educational Program for Cardiopulmonary Resuscitation Using Simman versus Traditional Manikin on 2nd Year Nursing Students' Performance IOSR. Journal of Nursing and Health Science, 6(1), 01-09.

Adams, J., Jose, R., Lauren, B., Patrick, G., Hughes, M., David, G., ... Michele, L. (2016). Management of Maternal Cardiac Arrest in the Third Trimester of Pregnancy: A Simulation-Based Pilot. Critical Care Research and Practice, 2016, 8. https://doi.org/10.1155/2016/5283765

Al Kandary, S., Al Jeheildi, A., Ghayath, T., \& Al Haid, N. (2012). Perceived competence in cardio-pulmonary resuscitation, knowledge and practice among qualified nurses in following cardiopulmonary resuscitation training: a review of the literature. Journal of Advanced Nursing, 51, 288-297.

Al-Foudri, H., Kevelighan, E., Catling, S., \& CEMACH. (2010). Saving mothers' lives: lessons for anaesthetists, Continuing Education in Anaesthesia. Crit Care Med., 10, 81-87.

Aly, A. (2010). Impact of a basic life support training program on nurses' knowledge and performance at emergency room. Doctorate thesis, Faculty of nursing, Suez Canal University.

Andreia de, A.R., Nuno C., \& Luís, M.G. (2014). Cardiac arrest in pregnancy: best practices are needed. Acta Obstet Ginecol Port, 8(2), 164-168.

Centre for Maternal and Child Enquiries (CMACE). (2006). Saving Mothers' Lives: reviewing maternal deaths to make motherhood safer. The Eighth Report on Confidential Enquiries into Maternal Deaths in the United Kingdom. BJOG 2011, 118(Suppl. 1), 201-203.

Cohen, S.E., Andes, L.C., \& Carvalho, B. (2008). Assessment of knowledge regarding cardiopulmonary resuscitation of pregnant women. Int $J$ Obstet Anesth, 17(1), 20-25. https://doi.org/10.1016/j.ijoa.2007.10.002

Damjan, L., Bojan, L., \& Jerneja, G. (2012). Impact ofadditional module training on the level of basic life support knowledge of first year student at theuniversity of Maribor ternational. Journal of Emergency Medicine, 4, 16.

Demestiha, D., Pantazopoulos, N., \& Xanthos, T. (2012). Use of the impedance threshold device in cardiopulmonary resuscitation. World Journal of Cardiology, 2(2), 19-26. https://doi.org/10.4330/wjc.v2.i2.19

Einav, S., Matot, I., Berkenstadt, H., et al. (2008). A survey of labourward clinicians' knowledge of maternal cardiac arrest and resuscitation. Int $J$ Obstet Anesth, 17(3), $238-242$. https://doi.org/10.1016/j.ijoa.2008.01.015

Elazazay H.M., Abdelazez A.L., \& Elsaie, O.A. (2012). Effect of Cardiopulmonary Resuscitation Training Program on Nurses Knowledge and Practice Kuwait. Bull Alex Fac Med, 43(2). Hamilton R. Nurses knowledge and skill retention.

Hui, D., Morrison, L.J., Windrim, R., Lausman, A.Y., et al. (2011). The American Heart Association 2010 guidelines for the management of cardiac arrest in pregnancy: consensus recommendations on implementation strategies. J. Obstet Gynaecol Can., $\quad 33, \quad 858-863$. https://doi.org/10.1016/S1701-2163(16)34991-X

Hussain, M., \& Lyneham, J. (2009). Cardio-pulmonaryresuscitation knowledge among nurses who work inBahrain. International Journal of Nursing Practice, $294-302$. https://doi.org/10.1111/j.1440-172X.2009.01752.x

Institute of Medicine. (2015). Strategies to improve cardiac arrest survival: A time to act. Washington, DC, The National Academies Press.

Jeejeebhoy, F.M., Zelop, C.M., Lipman, S., et al. (2015). Cardiac Arrest in Pregnancy. A Scientific Statement From the American Heart Association. https://doi.org/10.1161/CIR. 0300

Lavonas, E.J., Drennan, I.R., Gabrielli, A., et al. (2015). American Heart Association Guidelines Update for Cardiopulmonary Resuscitation and Emergency Cardiovascular Care-Part 10: Special Circumstances of Resuscitation. Circulation, 132, 501-518. https://doi.org/10.1161/CIR.0000000000000264

Meissner, T., Kloppe, C., \& Hanefeld, C. (2012). Basic life supportskills of high school students before and afterresuscitation training: a longitudinal investigation. Scandia Journal of Trauma Rhesus 
EmergencyMedical, 20, 31. https://doi.org/10.1186/1757-7241-20-31

Micheal, D.D., Pantazopoulos, N., \& Xanthos, T. (2014). Use of the impedance threshold device in cardio pulmonary resuscitation. World Journal of Cardiology, 2(2), 19-26. https://doi.org/10.4330/wjc.v2.i2.19

Mustafa, M.A., \& Jaddoue, B.A. (2014). Assessment of Nurses' Knowledge towards Cardiopulmonary Resuscitation at Al-Najaf City's Teaching Hospital. Journal of Kufa for Nursing Science, 4(1).

Myerburg R.J., \& Castellanos. (2015). Cardiac Arrest and Sudden Cardiac Death-Chapter 41(10th ed.). In Bonow R.O., Mann, D.L., Zipes, D.P., \& Libby, P. (Eds.), Braunwald's Heart Disease: A Textbook of Cardiovascular Medicine. Oxford, UK. Elsevier.

Nagashima, K., Takahata, O., Fujimoto, A., \& Iwasaki, H. (2012). Investigation of nurses' knowledge and experience incardiopulmonary resuscitation. Resuscitation, 14(2), 104-107.

Neal, J.M., Mulroy, M.F., \& Weinberg, G.L. (2012). American Society of Regional Anesthesia and Pain Medicine checklist for managing version. Reg Anesth Pain Med, 37, 16-18.

Parajulee, S., \& Selvaraj, V. (2011). Knowledge of nurses towards cardiopulmonary resuscitation in a tertiary care teaching hospital in Nepal. Journal of Clinical and Diagnostic Research, 5(8), 1585-1588.

Reza, P.K., Amir, J., Arsalan, N., Afshin, A., Mohammad, K., Masoud, R., \& Mohammad, A. (2017, May-Jun). Assessment of Iranian Nurses and Emergency Medical Personnel in Terms of Cardiopulmonary Resuscitation Knowledge Based on the 2010 Guideline. Iran J Nurs Midwifery Res., 22(3), 184-189.

Rittenberger, J.C., Kelly, E., Jang, D., \& Greer, K.H. (2010). A. Successful outcome utilizing hypothermia after cardiacarrest in pregnancy: a case report. Crit Care Med, 36(4), 1354-1356. https://doi.org/10.1097/CCM.0b013e318169ee99

Robertson, L., Schumacher, G., Gosman, R., Kanfer, M.K., \& DeVita, M. (2009). Simulation-based crisis team training for multidisciplinary obstetric providers. Simulation in Healthcare, 4(2), 77-83. https://doi.org/10.1097/SIH.0b013e31819171cd

Tan, E.K., \& Tan, E.L. (2013). Alterations in physiology and anatomy during pregnancy. Best Pract Res Clin Obstet Gynaecol, 27, 791-802. https://doi.org/10.1016/j.bpobgyn.2013.08.001

Wong M.K., Morrison L.J., Qiu, F., et al. (2014). Trends in short- and long-term survival among out-of-hospital cardiac arrest patients alive at hospital arrival. Circulation, 130(21), 1883-1890. https://doi.org/10.1161/CIRCULATIONAHA.114.010633

\section{Copyrights}

Copyright for this article is retained by the author(s), with first publication rights granted to the journal.

This is an open-access article distributed under the terms and conditions of the Creative Commons Attribution license (http://creativecommons.org/licenses/by/4.0/). 